Pacific Journal of Mathematics

DISTRIBUTIVE LATTICES WITH A THIRD OPERATION
DEFINED 


\title{
DISTRIBUTIVE LATTICES WITH A THIRD OPERATION DEFINED
}

\author{
B. H. ARNOLD
}

1. Introduction. If $L$ is the direct union of two distributive lattices, one may define a new operation $*$ between any two elements $(a, b)$ and $(c, d)$ of $L$ by

$$
(a, b) *(c, d)=(a \cap c, b \cup d) .
$$

This operation $*$ is:

$P$ 1. Idempotent

$P 2$. Commutative

$P 3$. Associative

P4. Distributive with $*, \cup, \cap$ in all possible ways.

The main results of this paper are the following.

First (Theorem 16), this is essentially the only way in which an operation with properties $P 1-P 4$ can arise in a distributive lattice. That is, if $L$ is a distributive lattice with a binary operation $*$ having properties $P 1-P 4$, then $L$ is a sublattice of the direct union of two distributive lattices, and the operation $*$ is given by equation (1).

Second (Theorem 9), if

$$
\text { P5. } L \text { contains an identity element } e \text { for the operation } * \text {, }
$$

then $L$ is the entire direct union. Here $P 5$ is sufficient but not necessary; a necessary and sufficient condition is given in Theorem 17. In case $*$ is identical with $U$ or $\cap$, Theorems 9, 16, and 17 still hold, but give trivial decompositions.

Finally, Section 5 shows that the presence of an operation $*$ is equivalent to the existence of a partial ordering with certain properties, so that our theorems may be restated so as to apply to distributive lattices with an auxiliary partial ordering.

2. Preliminary considerations. Throughout the paper, $L$ is a distributive lattice with an operation $*$ having at least properties $P 1-P 4$. By an isomorphism

Received February 10, 1950. Published with the approval of the Oregon State College Monographs Committee, Research Paper No.152, Department of Mathematics, School of Science.

Pacific J. Math. 1 (1951), 33-41. 
between two such algebraic structures, we shall mean a one-to-one correspondence which preserves the operations $U, \cap, *$; as is customary, in the direct union $A \times B$ of two such algebraic structures, all operations act coordinatewise; for example, $(a, b) *(c, d)=(a * c, b * d)$.

For later reference, we collect here several simple consequences of $P 1-P 4$. The proofs consist of repeated applications of the idempotent and other laws, and will be presented briefly and without annotation of the separate steps. These results will be used frequently in later proofs without any explicit reference being made. In these theorems, small Latin letters represent arbitrary elements of $L$.

THEOREM 1. $x \cap y \leq x$ * $y \leq x \cup y$.

Proof. We have

$$
(x \cap y) \cup(x * y)=[(x \cap y) \cup x] *[(x \cap y) \cup y]=x * y ;
$$

thus $x \cap y \leq x * y$. Similarly for the other inequality.

THEOREM 2. If $x_{1} \leq x_{2}$ and $y_{1} \leq y_{2}$, then $x_{1} * y_{1} \leq x_{2} * y_{2}$.

Proof. We have

$$
\left(x_{1} * y_{1}\right) \cap\left(x_{1} * y_{2}\right)=x_{1} *\left(y_{1} \cap y_{2}\right)=x_{1} * y_{1} ;
$$

thus $x_{1} * y_{1} \leq x_{1} * y_{2}$. Similarly, $x_{1} * y_{2} \leq x_{2} * y_{2}$, and the theorem follows.

THEOREM 3. $x *(x \cup y)=x \cup(x * y)$ and $x *(x \cap y)=x \cap(x * y)$.

Proof. Clearly,

$$
x *(x \cup y)=(x * x) \cup(x * y)=x \cup(x * y) .
$$

Similarly for the other equation.

THEOREM 4. $x$ * $y=(x \cap y) *(x \cup y)$.

Proof. The result follows from the continued equation,

$$
\begin{aligned}
(x \cap y) *(x \cup y) & =[(x \cap y) * x] \cup[(x \cap y) * y] \\
& =[(x * y) \cap x] \cup[(x * y) \cap y] \\
& =(x * y) \cap(x \cup y)=x * y .
\end{aligned}
$$

THEOREM 5. $x *(x * y)=x * y$.

Proof. Clearly,

$$
x *(x * y)=(x * x) * y=x * y \text {. }
$$


THEOREM 6. If $x \leq u \leq x$ * $y \leq v \leq y$, then $u$ * $v=x$ * $y$.

Proof. Since $x \leq u$ and $x * y \leq v$, Theorem 2 shows that

$$
x * y=x *(x * y) \leq u * v .
$$

Similarly,

$$
u * v \leq(x * y) * y=x * y .
$$

3. The operation $*$ has properties $P 1-P 5$. In this section, we prove one of the main results of the paper (Theorem 9), using the assumption that $L$ contains an element $e$ which is an identity element for the operation $*$.

THEOREM 7. If $a \leq e$ and $c \leq e$, then $a * c=a \cap c$.

Proof. Since $a * c=(a \cap c) *(a \cup c)$, it is sufficient to consider the case $a \leq c \leq e$ and prove $a * c=a$. But then $a \leq a \leq a * e \leq c \leq e$, and Theorem 6 shows that $a * c=a * e=a$.

THEOREM 8. If $b \geq e$ and $d \geq e$, then $b$ * $d=b \cup d$.

The proof is similar to that of Theorem 7 .

THEOREM 9. If $L$ is a distributive lattice with a binary operation * having properties $P 1-P 5$, then $L$ is isomorphic to the direct union of two distributive lattices $A, B$ each with an operation $*$ having properties P1-P5; and if $(a, b),(c, d)$ are any two elements of $A \times B$, then $(a, b) *(c, d)=(a \cap c, b \cup d)$.

Proof. Set

$$
A=\{a \mid a \leq e\}, \quad B=\{b \mid b \geq e\} ;
$$

then, with the same operations as in $L, A$ and $B$ are distributive lattices each with an operation $*$ having properties $P 1-P 5$.

We prove that the correspondence $(a, b) \rightarrow a * b$ is the required isomorphism from $A \times B$ onto $L$. It is clearly a single valued correspondence from $A \times B$ into $L$. It covers $L$ because, for any element $x$ of $L$, we have $x \cap e \in A, x \cup e \in B$ and, by Theorem 4, $(x \cap e) *(x \cup e)=x * e=x$. It is one-to-one because, for any $a \in A, b \in B$, we have $e \cap(a * b)=(e \cap a) *(e \cap b)=a * e=a$. Thus if $a * b$ $=c * d, c \in A, d \in B$, then $a=c$. Similarly, $b=d$.

This correspondence preserves the three operations $U, \Pi, *$. For instance, $(a, b) \cup(c, d)=(a \cup c, b \cup d) \rightarrow(a \cup c) *(b \cup d)=(a \cup c) *[(b \cup d) *(b \cup d)]$.

By Theorem 8, $b \cup d=b * d$; making this replacement in one parenthesis only, 
and rearranging the factors connected by $*$, we have $[(a \cup c) * b] *[(b \cup d) * d]$. But $b=b \cup c, d=a \cup d$, so that we have

$$
\begin{aligned}
& {[(a \cup c) *(b \cup c)] *[(b \cup d) *(a \cup d)]} \\
& \quad=[(a * b) \cup c] *[(b * a) \cup d]=(a * b) \cup(c * d),
\end{aligned}
$$

whence the operation $U$ is preserved by our correspondence. Similarly for $\cap$.

For the operation $*$,

$$
(a, b) *(c, d)=(a * c, b * d) \rightarrow(a * c) *(b * d)=(a * b) *(c * d) .
$$

Thus our correspondence is an isomorphism.

By 'Theorems 7 and $8,(a, b) *(c, d)=(a \cap c, b \cup d)$. This completes the proof.

REMARK. The element $e$ will be the $l$ in $A$ and the $O$ in $B$. The lattice $A$ will have an $O$ if and only if $L$ has one; $B$ will have an $I$ if and only if $L$ has one.

4. The operation $*$ has properties P1-P4. In this section, we prove one of the main results of the paper (Theorem 16). The method employed is to complete $L$ in such a way that $*$ has properties Pl-P5 and then to apply Theorem 9. Several preliminary definitions and theorems will be of use.

DEFINITION 1. We extend the operations $\cup, \cap$, * to act on any subsets $H, K$ of $L$ by defining $H \cup K=\{x \cup y \mid x \in H, y \in K\}$, and similarly for the other operations.

Notice that $H \cup K$, for example, is a subset of $L$, and is usually neither the supremum of the elements in the subsets $H$ and $K$ nor the point set union of $H$ and $K$.

DEFinition 2. A subset $P$ of $L$ is a $*$-ideal if $P * L \subset P$.

For any fixed $a \in L$, the set $a * L$ is a a-ideal; it is called the principal *-ideal generated by $a$.

THEOREM 10. An element $x$ of $L$ is in the principal $*$-ideal $A$ generated by $a$ if and only if $a * x=x$.

The sufficiency is evident. To prove necessity, we note that if $x \in A$, then $x=a * y$; by Theorem 5 it follows that $a * x=a *(a * y)=x$.

DEFINITION 3. A subset $H$ of $L$ is intervally closed if $x \in H, y \in H$, and $x \leq z \leq y$ imply $z \in H$. The interval closure of a set $G$ is the smallest intervally closed subset containing $G$.

It is easily seen that the interval closure of any set is the collection of elements which lie between two elements of the set. 
DEFINITION 4. A subset $R$ of $L$ is special if it is

(a) a *⿻一𠃋十deal,

(b) a sublattice, and

(c) intervally closed.

THEOREM 11. Each principal *-ideal is also a special subset.

Proof. Let $A$ be the principal * $*$-ideal generated by $a$, and let $x, y$ be any two elements of $A$. Then $a *(x \cup y)=(a * x) \cup(a * y)=x \cup y$, and $x \cup y \in A$, by Theorem 10. Similarly $x \cap y \in A$, and $A$ is a sublattice of $L$.

If $x, y$ are any two elements of $A$, and $x \leq z \leq y$, we must show that $z$ is in $A$. Since $A$ contains $a \bigcap x$ and $a \cup y$, there is no loss in generality in supposing that $x \leq a \leq y$. Set $a *(a \cup z)=u$. We prove first that $u=a \cup z$. Since $a * y=y$ and $a \leq u \leq y$, Theorem 6 shows that $u * y=y$, so that

$$
(a \cup z) \cap(u * y)=(a \cup z) \cap y=a \cup z .
$$

By Theorem 5, $u *(a \cup z)=u$. From the definition of $u$ and Theorem 1, we have $u \leq a \cup z$, so that

$$
[(a \cup z) \cap u] *[(a \cup z) \cap y]=u *(a \cup z)=u \text {. }
$$

But, from the distributive law, the left-hand members of equations (2) and (3) are equal, and $u=a \cup z$.

We now proceed with the proof that $z \in A$. Set

$$
v=z \cap(a * z)=z *(a \cap z) \text {. }
$$

Then, by Theorem 1, $a \cap z \leq v \leq z$, and

$$
a \cup v=a \cup[z *(a \cap z)]=(a \cup z) *[a \cup(a \cap z)]=(a \cup z) * a=u=a \cup z \text {. }
$$

But now

$$
v=(a \cap z) \cup v=(a \cup v) \cap(z \cup v)=(a \cup z) \cap(z \cup v)=(a \cup z) \cap z=z .
$$

That is, $z \cap(a * z)=z$, whence $z \leq a * z$. Similarly, $z \geq a * z$. Thus $z=a * z$ and, by Theorem 10, $z \in A$.

THEOREM 12. If $P$ is any *-ideal, the interval closure of the sublattice generated by $P$ is a special subset of $L$.

Proof. Let $Q$ be the sublattice generated by $P$, and let $\bar{Q}$ be the interval closure of $Q$. Then evidently $\bar{Q}$ is intervally closed.

$\bar{Q}$ is a sublattice because if $x, y \in \bar{Q}$, there exist elements $u_{1}, u_{2}, v_{1}, v_{2}$ of $Q$ such that $u_{1} \leq x \leq v_{1}, u_{2} \leq y \leq v_{2}$. Then $u_{1} \cup u_{2} \leq x \cup y \leq v_{1} \cup v_{2}$ and, since $Q$ 
is a lattice, $x \cup y \in \bar{Q}$. Similarly $x \cap y \in \bar{Q}$, so that $\bar{Q}$ is a sublattice of $L$.

$\bar{Q}$ is a $*$-ideal. Since $*$ distributes over $U, \cap, Q$ is a $*$-ideal, and Theorem 2 then shows that $\bar{Q}$ is a $*$-ideal. This completes the proof.

REMARK. It is evident that any special subset containing $P$ must contain $\bar{Q}$. Thus Theorem 12 gives a construction for the smallest special subset containing (generated by) a given *-ideal.

THEOREM 13. If $R, S$ are special subsets of $L$, then $R * S, R \cup S, R \cap S$ are special subsets of $L$.

Proof. That each of the sets $R * S, R \cup S, R \cap S$ is a *-ideal is a simple consequence of the distributive laws and Definition 1 .

To see that $R * S$ is a special subset, note that $R * S$ is contained in both $R$ and $S$, since both are *-ideals; but clearly $R * S$ contains the point-set intersection of $R$ and $S$ since $*$ is idempotent. Thus $R * S$ is this intersection, which is easily seen to be a special subset of $L$.

$R \cup S$ is intervally closed because, if $r_{1} \cup s_{1} \leq x \leq r_{2} \cup s_{2}$, then

$$
r_{1} \cap r_{2} \leq x \cap r_{2} \leq r_{2}
$$

and, since $R$ is a special subset, $x \cap r_{2} \in R$. Similarly, $x \cap s_{2} \in S$. But then

$$
\left(x \cap r_{2}\right) \cup\left(x \cap s_{2}\right)=x \cap\left(r_{2} \cup s_{2}\right)=x
$$

lies in $R \cup S$, and $R \cup S$ is intervally closed.

$R \cup S$ is a sublattice of $L$ because, if $r_{1} \cup s_{1}, r_{2} \cup s_{2}$ are any two elements of $R \cup S$, clearly $\left(r_{1} \cup s_{1}\right) \cup\left(r_{2} \cup s_{2}\right)=\left(r_{1} \cup r_{2}\right) \cup\left(s_{1} \cup s_{2}\right)$ lies in $R \cup S$. Also, since $r_{1} \cap r_{2} \leq\left(r_{1} \cup s_{1}\right) \cap\left(r_{2} \cup s_{2}\right)$ and $s_{1} \cap s_{2} \leq\left(r_{1} \cup s_{1}\right) \cap\left(r_{2} \cup s_{2}\right)$, we have

$$
\left(r_{1} \cap r_{2}\right) \cup\left(s_{1} \cap s_{2}\right) \leq\left(r_{1} \cup s_{1}\right) \cap\left(r_{2} \cup s_{2}\right) \leq\left(r_{1} \cup r_{2}\right) \cup\left(s_{1} \cup s_{2}\right) \text {. }
$$

But the two extreme elements of this sequence of inequalities lie in $R \cup S$; thus, since $R \cup S$ is intervally closed, the center element also lies in $R \cup S$. This completes the proof that $R \cup S$ is a special subset; dually, $R \cap S$ is a special subset.

DEFINITION 5. $\mathcal{L}=\{R, S, T, \cdots\}$ is the collection of all special subsets of $L$ with the three operations $U, \cap$, *

THEOREM 14. The set $\mathcal{L}$ with the operations $U, \cap$ is a distributive lattice and $*$ has properties Pl-P5.

Proof. Theorem 13 shows that $\mathcal{L}$ is closed under the operations $\cup, \cap$, * To show that $\mathcal{L}$ is a distributive lattice, we prove 
1. $R \cup R=R, R \cap R=R$,

2. $R \cup S=S \cup R, R \cap S=S \cap R$,

3. $(R \cup S) \cup T=R \cup(S \cup T),(R \cap S) \cap T=R \cap(S \cap T)$,

4. $R \cup(R \cap S)=R$,

5. $R \cup(S \cap T)=(R \cup S) \cap(R \cup T)$.

Numbers 1,2, and 3 are evident. To prove 4, we note that clearly

$$
R \cup(R \cap S) \supset R
$$

we show that $R \cup(R \cap S) \subset R$. If $x=r_{1} \cup\left(r_{2} \cap s\right)$ is any element of $R \cup(R \cap S)$, then $r_{1} \leq x \leq r_{1} \cup r_{2}$, and $x \in R$.

To prove 5, we note that clearly (R $\cup S) \cap(R \cup T) \supset R \cup(S \cap T)$; we show that $(R \cup S) \cap(R \cup T) \subset R \cup(S \cap T)$. If $x=\left(r_{1} \cup s\right) \cap\left(r_{2} \cup t\right)$ is any element of $(R \cup S) \cap(R \cup T)$, then $\left(r_{1} \cap r_{2}\right) \cup(s \cap t) \leq x \leq\left(r_{1} \cup r_{2}\right) \cup(s \cap t)$, and

$$
x \in R \cup(S \cap T) \text {. }
$$

The proofs that the operation $*$ has properties $P 1-P 4$ are similar to those just given and will be omitted. For $P 5$, the lattice $L$ itself is a special subset of $L$ and acts as the identity element for the operation $*$ in $\mathcal{L}$.

THEOREM 15. The correspondence $x \rightarrow$ the principal $*$-ideal generated by $x$ is an isomorphism of $L$ onto a sublattice of $\mathcal{L}$ which identifies the operations 米 in $L$ and the sublattice of $\mathcal{L}$.

Proof. By Theorem 10, if $x, y$ generate the same principal *-ideal, then $y=x * y=y * x=x$, so that the above correspondence is one-to-one.

To prove that this correspondence is an isomorphism, let

$$
x \rightarrow X=x * L, y \rightarrow Y=y * L ; \text { then } x \cup y \rightarrow(x \cup y) * L=Z \text {. }
$$

Clearly $Z \subset X \cup Y$. Conversely, if $w=(x * u) \cup(y * v)$ is any element of $X \cup Y$, then $(x \cup y) *(u \cap v) \leq w \leq(x \cup y) *(u \cup v)$, and $w \in Z$. The proofs for $\cap$, * are similar, and will be omitted.

Theorems 9, 14, and 15 give immediately our main result:

THEOREM 16. If $L$ is any distributive lattice with an operation $*$ having properties P1-P4, then $L$ is isomorphic to a sublattice of the direct union of two distributive lattices $A, B$, each with an operation $*$ having properties P1-P5; and if $(a, b),(c, d)$ are any two elements of $A \times B$, then

$$
(a, b) *(c, d)=(a \cap c, b \cup . d) .
$$

THEOREM 17. If $L$ is any distributive lattice with an operation $*$ having properties $P 1-P 4$, then $L$ is isomorphic to a direct union in which the operation $*$ 
is given by equation (1) if and only if each pair of elements of $L$ is contained in some principal $*$-ideal.

Necessity. If $L$ is a direct union with $*$ given by equation (1), the two arbitrary elements $(a, b),(c, d)$ are contained in the principal $*$-ideal generated by $(a \cup c$, $b \cap d)$.

Sufficiency. By Theorem 16, $L$ may be considered as a sublattice of a direct union in which $*$ is given by equation (1). Let $\left(x_{1}, y_{1}\right)$ be any fixed element of $L$, and define $A=\left\{x \mid\left(x, y_{1}\right) \in L\right\}, B=\left\{y \mid\left(x_{1}, y\right) \in L\right\}$; then $A \times B \subset L$. In fact, if $\left(x, y_{1}\right)$ and $\left(x_{1}, y\right)$ are in the principal $*$-ideal generated by $(a, b)$, then

$$
a \geq x \cup x_{1}, b \leq y \cap y_{1},
$$

and $L$ contains

$$
\left[\left(x, y_{1}\right) \cap(a, b)\right] *\left[\left(x_{1}, y\right) \cdot \cup(a, b)\right]=(x, b) *(a, y)=(x, y) .
$$

Conversely, $L \subset A \times B$, for if $(x, y)$ is any element of $L$, and $(a, b)$ generates a principal $*$-ideal containing $(x, y)$ and $\left(x_{1}, y_{1}\right)$, then $L$ contains

$$
\begin{aligned}
& {[(x, y) \cap(a, b)] \cup\left\{\left(x_{1}, y_{1}\right) \cap\left[(x, y) *\left(x_{1}, y_{1}\right)\right]\right\}} \\
& \quad=(x, b) \cup\left\{\left(x_{1}, y_{1}\right) \cap\left(x \cap x_{1}, y \cup y_{1}\right)\right\}=\left(x, y_{1}\right) .
\end{aligned}
$$

Similarly, $\left(x_{1}, y\right)$ is in $L$, and $(x, y) \in A \times B$.

CAUTION. The decomposition of $L$ will be trivial (one of $A, B$ consisting of a single element) if and only if $*$ is identical with $U$ or $\cap$.

5. The ordering equivalent to $*$. In any distributive lattice $L$ with an operation * having properties $P 1-P 4$, we may define an auxiliary order relation by making $x \succ y$ mean $x * y=y$. It is easily seen that this order relation has the following properties:

O1. $x>x$;

02. $x \succ y, y \succ x$ imply $x=y$;

03. $x \succ y, y \succ z$ imply $x \succ z$;

O4. Any two elements $x, y$ of $L$ have a greatest lower bound (namely $x * y)$;

O5. The operation of taking the greatest lower bound is distributive with itself and with the two lattice operations in all possible ways. 
Conversely, if $L$ is any distributive lattice (with no additional operation $*$ defined) with an auxiliary order relation having properties $01-05$, then the operation * defined in $L$ by setting $x$ * $y$ equal to the greatest lower bound of $x$ and $y$ has properties $P 1-P 4$. Moreover, the operation $*$ will have property $P 5$ if and only if the order relation satisfies:

O6. There is a greatest element $e$ in $L$.

Our results may thus be restated as theorems concerning distributive lattices with an auxiliary order relation.

\section{REFERENCES}

1. G. Birkhoff, Lattice theory, Amer. Math. Soc. Colloguium Publications, vol.25; American Mathematical Society, New York, 1948. See especially p. 92, Theorem 9.

2. Neutral elements in general lattices, Bull. Amer. Math. Soc. 46 (1940), 702-705.

3. C. J. Everett and S. Ulam, Projective algebra I, Amer. J. Math. 68 (1946), 77-88.

4. F. Klein-Barmen, $\ddot{U}$ ber Verbande mit einer weiterer assoziativen und kommutativen Elementverknupfung., Math. Z. 47 (1940), 85-104.

5. A. Stöhr, Über zweifach geordnete mengen und Zerlegungen in Rechtecke I, J. Reine Angew. Math. 184 (1942), 138-157.

6. P. M. Whitman, Splittings of a lattice, Amer. J. Math. 65 (1943), 179-196.

Oregon State College 



\title{
EDITORS
}

\author{
HERBERT BUSEMANN \\ University of Southern California \\ Los Angeles 7, California
}

R. M. RoBINSON

University of California

Berkeley 4, California

E. F. BECKENBACH, Managing Editor

University of California

Los Angeles 24, California

ASSOCIATE EDITORS
R. P. DILWORTH
P. R. HALMOS
BQRGE JESSEN
J. J. STOKER
HERBERT FEDERER
HEINZ HOPF
PAUL LÉVY
E. G. STRAUS
MARSHALL HALL
R. D. JAMES
GEORGE PÓLYA
KÔSAKU YOSIDA

\section{SPONSORS}

UNIVERSITY OF BRITISH COLUMBIA

CALIFORNIA INSTITUTE OF TECHNOLOGY UNIVERSITY OF CALIFORNIA, BERKELEY

UNIVERSITY OF CALIFORNIA, DAVIS

UNIVERSITY OF CALIFORNIA, LOS ANGELES

UNIVERSITY OF CALIFORNIA, SANTA BARBARA

OREGON STATE COLLEGE

UNIVERSITY OF OREGON
UNIVERSITY OF SOUTHERN CALIFORNIA STANFORD UNIVERSITY

WASHINGTON STATE COLLEGE

UNIVERSITY OF WASHINGTON

AMERICAN MATHEMATICAL SOCIETY NATIONAL BUREAU OF STANDARDS, INSTITUTE FOR NUMERICAL ANALYSIS

\section{Vari-Type Composition by \\ Cecile Leonard \\ Ruth Stafford}

With the cooperation of

E. F. Beckenbach

E. G. Straus

Printed in the United States of America by

Edwards Brothers, Inc., Ann Arbor, Michigan

UNIVERSITY OF CALIFORNIA PRESS - BERKELEY AND LOS ANGELES COPYRIGHT 1951 BY PACIFIC JOURNAL OF MATHEMATICS 


\section{Pacific Journal of Mathematics}

\section{Vol. 1, No. $1 \quad$ November, 1951}

Ralph Palmer Agnew, Ratio tests for convergence of series............. 1

Richard Arens and James Dugundji, Topologies for function spaces....... 5

B. Arnold, Distributive lattices with a third operation defined ........... 33

R. Bing, Concerning hereditarily indecomposable continua ........... 43

David Dekker, Generalizations of hypergeodesics ............... 53

A. Dvoretzky, A. Wald and J. Wolfowitz, Relations among certain ranges of vector measures.................................... 59

Paul Erdős, F. Herzog and G. Pirani, Schlicht Taylor series whose convergence on the unit circle is uniform but not absolute .......... 75

Whilhelm Fischer, On Dedekind's function $\eta(\tau) \ldots \ldots \ldots \ldots \ldots \ldots . \ldots 3$

Werner Leutert, The heavy sphere supported by a concentrated force ...... 97

Ivan Niven and H. Zuckerman, On the definition of normal numbers ...... 103

L. Paige, Complete mappings of finite groups .................. 111

Otto Szász, On a Tauberian theorem for Abel summability ............. 117

Olga Taussky, Classes of matrices and quadratic fields ............. 127

F. Tricomi and A. Erdélyi, The asymptotic expansion of a ratio of gamma functions .................................... 133

Hassler Whitney, On totally differentiable and smooth functions ......... 143 